\title{
Balancing Immunity in a Setting of Covid-19
}

\author{
James Paul Pandarakalam*
}

Northwest Boroughs Healthcare NHS Foundation Trust, AFG Hospitals, Warrington WA2 $8 \mathrm{WN}, \mathrm{UK}$

ISSN: 2578-0093

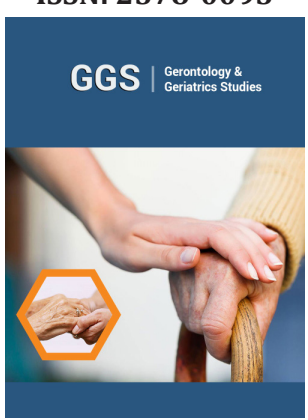

*Corresponding author: Dr James Paul Pandarakalam, Northwest Boroughs Healthcare NHS Foundation Trust, Hollins Park Hospital \& AFG Rehab Hospitals, Warrington WA2 8WN, UK, Email: James. pandarakalam@nwbh.nhs.uk

Submission: 海 July 23, 2020

Published: 悳 August 12, 2020

Volume 6 - Issue 2

How to cite this article: James Paul Pandarakalam. Balancing Immunity in a Setting of Covid-19. Gerontol \& Geriatric stud. 6(2). GGS. 000632. 2020.

DOI: $10.31031 /$ GGS.2020.06.000632

Copyright@ James Paul Pandarakalam, This article is distributed under the terms of the Creative Commons Attribution 4.0 International License, which permits unrestricted use and redistribution provided that the original author and source are credited.

\begin{abstract}
Only precautionary measures are practical at the moment in controlling the COVID-19 pandemic and one such measure is balancing self-immunity. The recent findings about the durability of COVID-19 antibodies in the infected people has put some concerns about the prospects of long-term immunisation methods. In such circumstances, one way of protecting from COVID-19 is by enhancing one's own immune mechanisms. Unfortunately, there is less discussion about this serous issue. Elderly patients are at a higher risk of getting infected with SARS-CoV-2 and should be mindful of balancing the immunity. Adherents of Integrated medicine seem to have more awareness of different facets of immunity and they go beyond the conventional methods. Immunological research is in a standstill. This paper is an attempt to evaluate balancing immunity through all the known practices done in different disciplines of medicine.
\end{abstract}

Keywords: Immunity; Diet; Sleep; Exercise; Destressing; Abstinence; Homeostasis

\section{Introduction}

The interconnectedness and the intricacies of human immune system is still unclear. Research in immunity could be clinically and experimentally cantered and involves study of fundamental biology of both the innate and adaptive immune response, immunity in infectious diseases, autoimmunity, rheumatology and transplantation immunology. Metaphorically, immune system is compared to a fortified building structure or a castle surrounded by a fortress and security system of hierarchical order; the fortress and the ground security system represent the innate immunity and the rest stand for the adapted immunity. Immunity is not absence of a specific disease or illness; rather, it is a balanced physiological and psychological state, the most sophisticated and elegant system of human physiology.

COVID-19 poses great immunological challenges to everybody on this planet. From genomic surveillance of clinical samples from patients with viral pneumonia in Wuhan, China, a novel coronavirus (termed 2019-nCoV) has been recognized [1]. A phylogenetic analysis of 2019-nCoV, sequenced from nine patients' samples, showed that the virus belongs to the subgenus Sarbecovirus [2]. The same study identified that Covid-19 was more similar to two bat-derived coronavirus strains, bat-SL-CoVZC45 and bat-SL-CoVZXC21, than to known human-infecting coronaviruses, including the virus that caused the SARS outbreak of 2003. SARS-CoV-2 belongs to the same large family of viruses as SARS-CoV, known as coronaviruses. Common symptoms include fever, dry cough, shortness of breath, loss of smell, taste and the disease can progress to pneumonia in severe cases and other serious complications. The disease caused by SARS-CoV-2, called COVID-19, has an estimated mortality rate of about $2.3 \%$. People who are older or have underlying health conditions seem to be most at risk of having severe disease or complications. As of 25 July 2020, more than 15.7 million cases have been reported across 188 countries and territories, resulting in more than 639,000 deaths. COVID-19, the viral disease does not discriminate people at a social or national level, but it seems to discriminate people at a genetic and immunity level.

\section{Immune system}

Unlike innate immunity, which generally involve leukocytes, adaptive immunity is controlled by the lymphocytes of two categories known as B lymphocytes and T lymphocytes. 
If $\mathrm{B}$ lymphocytes are the antibody producers, $\mathrm{T}$ lymphocytes are the master controllers. Natural killer (NK) cells recognize changes in our own cells and swiftly respond to kill our own cells when they get infected with virus. Our genetic code differs from each other only by one percent of the 25000 genes. The gene cluster which plays a large part in our health is called the human leukocyte antigen (HLA), also known as the major histocompatibility complex (MHC). Unique combination of compatibility genes determines immunological individuality. Their differences have positive and negative value. It is because they are different from individual to individuals, pandemics of the past could not wipe out human race. In the same way, it is this genetic identity responsible for organ rejection and transplant cases have to deal with the lifelong challenges of tissue rejection. Compatibility genes do not give protection for the individual from all infections. It might protect a person from one family of viruses only to be infected with another one. Compatibility genes cannot fix our immunity from alterations. It is continuously fostered by human life experiences, environmental changes, and lifestyles. It is an internal sensory system; it can sense and respond to environmental, hormonal, nutrient and even brain signals [3].

\section{Enrichment of immunity}

Vaccines are pathogen-specific, and they do not bestow an overall balanced immunity. Vaccine could be only a short-term solution if the antibody level declines after a short period. Safe immunisation, effective medications to block the cytokine storm responsible for the complications of COVID-19 are sure ways of combatting the current pandemic, but these might take some time to come into realty. There are many myths surrounding immunity enhancement. Enrichment of the immune system is possible so that it becomes vigilant and active in the event of an invasion by pathogens and it may possibly prevent immunity anomalies. Balancing of immunity can be achieved by focussing on healthy diet, moderate exercise, good sleep, restricting alcohol, free of smoking and destressing (Table 1).

Table 1: Influencing factors.

\begin{tabular}{|c|}
\hline Weight Monitoring \\
\hline Healthy Diet \\
\hline Supplements \\
\hline Moderate Exercise \\
\hline Sleep Hygiene \\
\hline Restricting Alcohol \\
\hline Substance Misuse \\
\hline Maintaining Healthy Lungs \\
\hline Thermal Balancing \\
\hline Managing Stress \\
\hline
\end{tabular}

According to the principles of integrated medicine, there may also be an extra-physiological immunity that can be brought into action in times of physiological crisis. One route to deal with the toxic pathogen is balancing our immunity. To defeat the tiger, one may need to become stronger than the tiger. To do this with COVID-19, we may need to foster our existing immune system, which can be done in many subtle ways. We all must grapple with the unprecedented threat posed by COVID-19, and frontline health workers must be mindful of their own immune systems when advising their patients to do the same. There are many myths surrounding immunity enhancement. The following methods offer some guidelines to balance immunity.

\section{Weight monitoring}

There is an inverse relationship between obesity and immunity. The same amount of similar vaccine administered to an obese person and a lean person generate different immune response. One of the modifiable risk factors of severe COVID-19 is reducing excess weight which will bring wider health benefits. Having a healthy body weight is an important factor in maintaining stronger immunity. Persistently enlarged fat cells mean that a person's body is always in a state of inflammation and the immune system is permanently on the go. Enough body fat is crucial for immunity and health.

Brown fat is composed of several small lipid droplets and a large number of iron-containing mitochondria. The purpose of brown fat is to burn calories in order to generate heat. White fat is composed of a single lipid droplet and has far less mitochondria and blood vessels, thus resulting in its lighter white or yellow appearance. White fat is the predominant form of fat in the body, originating from connective tissue. It provides the largest energy reserve in the body. White visceral fat is associated with metabolic syndrome that signal an increased risk for heart disease, diabetes, and cancer. Immunity plays a key role to help turn white fat into healthier brown fat. Because obese people have a weaker immune system, there is only a lower amount of white to brown fat conversion [4]. A strict dietary regime and moderate exercise are essential to maintain a healthy weight.

A recent public health study indicated that people who are overweight, with a BMI of 25 to 29.9, have a higher risk of hospitalization and poor outcomes if they get infected with SARSCoV-2 [5]. They discovered that having a BMI of 35 to 40 increases the risk of death from coronavirus by 40 per cent compared to people of healthy weight and a BMI of 40 or more increases the risk by 90 per cent. The report said being overweight or obese increases the chance somebody will end up critically ill in intensive care. It found that 7.9 per cent of critically ill patients with Covid-19 in intensive care units have a BMI of over 40 compared, with 2.9 per cent of the general population.

\section{Healthy diet}

Carbohydrates can suppress the immune system 30 minutes after they are consumed, and this effect may only go away after five hours. Carbohydrates cause inflammation, which has been shown to decrease immunity by weakening white blood cells. 
Too much fat cells suppresses immunity, and obesity can lead to a depressed immune system and can affect the body's ability to reproduce leucocytes, generate antibodies and locate infection sites. Pro-inflammatory foods can sabotage the immune system and should be avoided. Foods that have excessive diuretic properties have detrimental effects on the immune system, as it functions better when well-hydrated. Plenty of fruits and vegetables are recommended. A well-balanced diet is crucial to keep immunity levels high. An ideal immunity diet would maintain caloric balance and consist of healthy fats, phytonutrients and fibre with quality carbs and diverse protein sources. Many wellness products advertise quick immunity enhancement, but these claims have no scientific validity. In general, the Mediterranean dietary pattern has been praised as being anti-inflammatory and good for fortifying immunity [6].

\section{Supplements}

Dietary supplements and supplementary medicine may be required for people who suffer from micronutrient deficiencies. Vitamin D is linked with a healthy immune system, and a large amount of well-established data highlights the antiviral effects of vitamin $\mathrm{D}$, which can directly interfere with viral replication, but can also act in an immunomodulatory and anti-inflammatory manner [7-10]. Low levels of Vit D reduce our immune resistance against viruses [11]. A research study in U.S. claims that low levels of vitamin D cause twice the rate of death due to heart attack when compared with higher levels of vitamin D [12]. Everyone living in the UK should supplement with vitamin D between October and March to help maintain healthy levels over the darker months. Vitamin D supplements are available in several forms: capsules, sublingual sprays or liquid drops which are usually in an oil base as it is a fat-soluble vitamin. An over boosted immune system might lead to autoimmune reaction; what is needed is balancing and not over-boosting. Moreover, Vit D toxicity could lead to hypercalcemia and the excess calcium can get deposited in kidney, lungs, and heart.

Nearly all immune cells have vitamin D receptors, vitamin D networks with the immune system. Vitamin D supports to regulate both the innate and adaptive immune systems and is critical for balancing the immune function. Vit $\mathrm{D}$ has been indicated to reduce the production of pro-inflammatory cytokines that are associated with lung damage caused by acute viral respiratory infections such as influenza and Covid-19 [13]. Virtually all immune cells have vitamin D receptors, indicating vitamin D interacts with the immune system. Vitamin D is required to regulate both the innate and adaptive immune systems and its deficiency is associated with immune dysregulation. Many of the ways this vitamin affects the immune system are directly relevant to body's ability to defend against viruses. For example, Vit D triggers the production of cathelicidin and other defensins which are natural antivirals capable of preventing the virus from replicating and entering a cell. Vit D can also increase the number of CD8+ T cells, which play a critical role in clearing acute viral infections in the lungs. Vit D also suppresses pro-inflammatory cytokines and it is also proposed to come into play to alleviate the "cytokine storm" occurring in the most severe COVID-19 cases. This vitamin plays an essential role in glucose homeostasis, insulin sensitivity and regulation of adipokines such as leptin, as well as inflammatory cytokines [14].

Evidence from randomized controlled trials suggest regular vitamin D supplementation may help protect against acute respiratory infections. Admittedly, the direct evidence about the role of vitamin D in COVID-19 is still scanty. One small study from the United States and another study from Asia found a strong correlation between low vitamin D status and severe infection with COVID-19. It is now well recognized that the elderly and people with pre-existing conditions are more vulnerable to COVID-19. Notably, people with existing medical conditions are also often vitamin D deficient. Studies assessing ICU patients have reported low levels of vitamin D among them even before the development of COVID-19.

There is a higher incidence of COVID-19 reported among migrant people into cold weather from tropical countries (BAME people). BAME communities are prone to develop vitamin D deficiency because higher levels of melanin in their skin lead to lower levels of vitamin D absorption. Consequently, longer exposure to sunlight is required to accrue the equivalent quantity of the vitamin that may be produced in the white population. This is further exacerbated in colder countries like the U.K., which sees less sunlight and BAME persons spending more time indoors without giving a chance for the absorption of Vitamin D. So, there may be a connection between lower levels of the vitamin D in BAME communities and higher levels of COVID-19 cases, even though there is currently no firm data to demonstrate this alleged link.It appears logical to hypothesize a link between the high rates of COVID-19 infections in ethnic minority groups in the UK and US and the observed lower levels of vitamin D among them. Moreover, it is not possible to get sufficient supply of vitamin D through food alone and exposure to sunlight is indispensable.

\section{Moderate exercise}

Being active reduces stress and causes individuals to feel more energetic and alert, thereby helping the body to prepare for better sleep. The main principle of all exercise is to keep the body moving. Stress hormones are slowly released during exercise, and this has a favourable effect on the immune system [15]. Getting rid of bacteria in the pulmonary system may be facilitated by physical activity. The levels of white blood cells and antibodies can be changed by exercise. It is believed that during exercise, leucocytes and antibodies move faster in the circulatory system, so they can detect internal threats or diseases earlier; however, there is not yet any proof as to whether infections are prevented by these changes. Bacterial growth may be blocked by the increase in temperature during and after exercise. This temperature rise may help the body to fight infection, similar to what happens when someone has a 
fever in the course of the body's fight against infection, but these conjectures have not been fully proven. Maintaining a healthy basal metabolic rate is crucial.

\section{Sleep hygiene}

Sleep is the bedrock of immunity, as a lot of calibration happens when a person is sleeping that allows the immune system to work optimally. It is during sleep that the immune system heals and repairs and prepares for the challenges of the wakeful period. During the deep stages of NREM sleep, the body repairs and recuperates. The body produces certain chemicals that act on the brain, and the feeling of lethargy may be a signal from the body when fighting an infection, prompting the individual to sleep so that it can recover. Respiratory infection is linked to poor sleep [16]. A single night of poor sleep leads to a dramatic reduction in NK cells, the first line defence against viruses and cancerous cells, and this will have a negative impact on other immune cells. Those who get frequent colds are advised to check their sleep pattern [17]. Deep sleep reinforces immunological memories of previous pathogens [18].

\section{Restricting alcohol}

Alcohol has an immunosuppressant effect. Binge drinking is particularly detrimental to the immune system. One study found that after four shots of vodka drunk over a 20-minute period, blood samples revealed the immune systems of the participants ramped up at first, but after a few hours, their immune responses became sluggish. Acute and chronic alcohol use impedes cellular immune function and places binge drinkers at greater risk for bacterial and viral infections. A multi-layered interaction between alcohol and immunity exists, and alcohol abuse has a negative effect on innate and adaptive immunity [19]. Drinking alcohol immoderately can cause damage to the immune system in two ways. First, it reduces the overall available nutrients, thus depriving the body of valuable nutrients that foster the immune system. Second, excessive consumption of alcohol can hinder the ability of white blood cells to destroy microbes. It is well-recognized that the consumption of alcohol in excessive amounts can suppress white blood cell replication, inhibit the action of killer white cells on cancer cells and lessen the ability of macrophages to produce tumor necrosis factors. Damage to the immune system increases in proportion to the quantity of alcohol consumed. While wine promoters assert that one unit of red wine may be helpful to maintain health, any amount of alcohol that is enough to cause intoxication is also enough to suppress immunity.

\section{Substance misuse}

Marijuana, cocaine, heroin, and other opiates are widely used illegal drugs. Drug abuse compromises immunity, so it is imperative to stay clear of illicit drugs while a pandemic is going on. There have been numerous clinical reports on the association between infectious diseases and the use of illegal drugs. These drugs not only alter neurophysiological and pathophysiological responses, but also immunity responses. Thus, it is vital to determine the mechanisms by which drugs compromise immune responses in general and in concert with immunosuppressive viruses [20].

\section{Maintaining healthy lungs}

Smoking is linked with the negative progression and adverse outcomes of COVID-19 [21]. Smokers touch their lips and accidentally pass the virus to their mouth, and they tend to have existing respiratory conditions consequent to their smoking habit. These factors make them more vulnerable to a respiratory virus infection. Smokers are also prone to COVID-19-related complications. Smoking has been linked with adverse disease prognoses and a plethora of respiratory diseases [22]. Smokers are more vulnerable to infectious diseases because smoking is harmful to the immune system and affects how it responds to infections [23]. During the previous MERS-CoV epidemic, smokers were found to have high mortality rates [24]. In one retrospective analysis of 78 patients in China, smoking was correlated with more severe disease and a poorer prognosis of COVID-19. Even though there are conflicting analytical studies,[25] smoking continues to be linked with higher risk [26].

\section{Thermal balancing}

It is well accepted fact that fever is body's defence mechanism to activate the immune system in the event of inflammation. Our immune system functions at an optimum level in higher comfortable temperature. Immune system becomes underactive in cold weather and seasonal infectious diseases like influenza become more prevalent in lower temperature. There are some data to suggest that immune cells are more active in higher temperatures, and this conjecture is supported by the fact that fever due to infection is a mechanism of the body activated by the immune mechanism to defend against the pathogen. It has been established that if the body temperature is increased by $1{ }^{\circ} \mathrm{C}$ than normal, the immunity will instantly increase by 5 to 6 times. Likewise, if the temperature is reduced by $1{ }^{\circ} \mathrm{C}$ than normal, immunity will decrease by 5 to 6 times. This observation has some value in explaining the apparent higher incidence of COVID-19 infection and consequent mortality among the ethnic groups who were born and brought up in a hotter temperature and migrated to cold weather.

The temperature dependent immune response is linked with genetics. One probable explanation of higher incidence of COVID-19 among the BAME community may be that their immune cells are genetically wired to function better in hot weather and are not able to function at an optimum level in cold weather. Such a genetically determined immunological build-up means that their immune cells are slow to react to the viral invader. The immune cells of BAME persons are adapted to warm weather and are not as well-adapted to cold weather in comparison to the Caucasians who are born and brought up in the colder climates and adapted to lower temperature. 
The immune cells of BAME people may become underactive in cold weather. Even though the COVID-19 virus thrives equally well in hot and cold weather, BAME population have immunity shortcomings in surviving the cold winter months and this insight might prompt them in taking special precautions in future cold seasons.

Low temperature is recognized as being immunosuppressive. It has been observed that even cold-blooded animals migrate to warmer places when they become ill. An increase in body temperature has been long associated with infection and inflammation as a defense mechanism. Generation and differentiation of a particular kind of lymphocyte, known as a 'CD8+ cytotoxic T-cell' is enhanced by hyperthermia. Elevated body temperature changes the membranes of T-cells, which may help mediate the effects of micro-environmental temperature on cell function. Sub-thermoneutral laboratory housing temperature has been shown to induce immunosuppression in mice experiments and when the mice are housed at thermoneutral ambient temperature, a striking reduction in tumour formation, growth rate and metastasis has been observed [27]. Mice experiments with antigens demonstrated that mice who had a raised temperature due to antigen input showed a greater number of the type of CD8 T-cells that are capable of destroying infected cells $[28,29]$. Parallels were observed in the teleost fish [30]. Higher temperatures also seem to interfere with the replication of microbes. This is particularly seen when a host has a higher temperature and their immune system is temporarily enhanced as their temperature rises with a fever. The persistent cold weather of Hong Kong has been attributed as a cause of the rapid spread of SARS in 2003. BAME communities whose immune mechanisms are genetically evolved for survival in higher temperatures are compromised in the lower temperatures of the western countries. The cold weather puts additional stress on their immune cells which give into the viral invaders.

The above argument can be further supported by the spreading pattern of the seasonal flu. During the cold flu season, human immune cells become less efficient and flu viruses, which are unaffected by the low temperatures, are in an advantageous position to defeat the human immune cells. Such a hypothesis explains the higher incidence of flu in the winter period and challenges the misconception that the flu virus is killed by hot weather and thrives in cold weather. The Spanish flu that broke out in the United States in 1918 during winter seemed to ease off during the summer, but returned with a deadlier strain in the autumn, and a third wave the following year. The actual problem seems to on the human side, and viruses are unaffected by the seasonal temperature variations. One way of warming up the body is being metabolically active along with keeping up the room temperature or using protective clothes.

\section{Managing stress}

States of mind have long been associated with immune responses; severe anxiety suppresses our immune system, and the coronavirus may literally feed on fear. Current evidence illustrates that susceptibility to infections can be increased by chronic stress through suppression of the T-helper 1 immune response in favor of a T-helper 2 immune response [31]. Stress management, lifestyle changes, and career management could be considered to reduce susceptibility to infection. Relaxing and focusing on the present can aid in better mental health and counteract negative feelings. Different forms of meditation and progressive muscle relaxation techniques can help us unwind from the assault of day-to-day stressors. Relaxation after work may help to enhance the immune system.

Psychological health and immunity are causally related. Positivity strengthens our immune system. It is well proven that anxiety, hostility, and other negative states affect the immune system adversely. Nobody can evade stress, but we can modify our response to stress and protect the body from constant hormonal battle. In times of stress, brain sends defence signals to the endocrine system which in turn releases an array of hormonal chemicals that may solve the emergency, but prolonged exposure to them suppress the immune system leading to health issues [32]. As the pandemic is going on, we are all trapped to adjust to a new, rather strange way of life and this can be difficult for our mental wellbeing.

\section{Immunity and integrated medicine}

Unreasonable fear of COVID-19 only weakens the immune system, and fear attracts that which is feared. According to many COVID-19 survivors, remaining positive is a crucial factor in combatting this illness. Knowledge about the enemy and our potential resources lessens fears and helps us to plan strategies to defeat the adversary. With a quarter of the world's population in the grip of COVID-19, it is a highly challenging period to learn to survive and strengthen our body and mind and enhance our immune system, even using the wisdom of unconventional medicines and faith traditions. We will have to battle with this invisible enemy until an effective vaccine is identified. Anything that fosters selfimmunity should be encouraged in this time of a global medical emergency. Immunity is not a single entity; it is a system, and for a system to function well, it requires balance and harmony.

Not everything about the immune system is known to science, and according to integrated medicine, immunity may not be confined to physiology alone, but may have non-physiological aspects as well. Professor C.P. Mathew, an Indian oncologist, claims that he has successfully treated many cancer patients by integrating Allopathy and Siddha medicine (i.e., a form of alternative medicine). He is of the view that healing should ultimately be achieved by enhancing the immune system, and Siddha medicines are immune system stimulators. Energy medicines as a whole work along these lines. What is needed is a sympathetic understanding of complementary and alternative medicines and development of an integrated form 
of medicine. Studies of quantum bioenergy fields should be an integral part of the science of human physiology and homeostasis should be redefined as the state of steady internal physical and chemical conditions maintained by different regulators, including extrasomatic energy fields [33].

Complementary medicines work on the assumption that humans are associated with a subtle energy system, in addition to their material body. Even though such extrasomatic energy systems are not recognized in the modern medical sciences, there are energy fields that cannot be explained by the classic MaxwellSchrodinger equation. Most interactions between cells occur at a speed greater than the speed of light, so some have argued that cells must communicate through a quantum field in which the linear, predictable characteristics of the Newtonian world do not exist [34]. Analogous to the fact that earth has invisible undercurrents, biological systems may have unphysical energy networks, which may only be unphysical with present-day instrumentations. This force or energy field is a barrier made up of energy, plasma or particles that protect a person from environmental attacks and intrusions.

Humans are multidimensional or psycho-spiritual entities with several layers of energy bodies with increasing subtilty [35]. The materialbodyandenergybodiesareina complementaryrelationship: if the material body is the container, vital energy is the content [36]. Beverly Rubik postulated that biological systems may be regarded as complex, non-linear, dynamic, self-organising systems of energy and field phenomena $[37,38]$. Many researchers have attempted to bring the existence of extrasomatic energy fields into the arena of mainstream sciences [39-42]. If such quantum bioenergy fields really exist, they may play a major role in maintaining homeostasis in the human physiology, and it would be of great clinical interest to evaluate their role in immune system functionality, as long as they do not overrun the scientifically accepted views. To bring the concept of extra-physiological immunity into immunology, we may also have to accept the possibility of 'nano immune cells' and a 'nano-level immune mechanism'. To use a military analogy, the classical physiological immunity becomes the ground force and the extra-physiological immunity turns into the top military hierarchy. Such novel concepts of theoretical physiology warrant further exploration and elaboration.

Alternative medicine takes into account the friendly bacterial flora inhabiting human body and to be mindful of their role in balancing immunity. There are several trillions of bacteria existing within our body and gut is considered as the front yard of the bacterial colony. Even though humans are controlled by 25000 genes, the genes of the microbes cohabiting with ours are taken into account, it would be more than 3 million. In fact, these genes of the microbes are the immunity trainers and mentors of human immune cells [43]. Gut is the front yard of our microbial flora. Gut health is the meeting point of scientific medicine and alternate medicines because both acknowledge the existence of friendly gastrointestinal microbes [43]. A British scientist lived with a hunter's tribe in Africa for months following their lifestyle and dietary habits. When he examined his stool afterwards, he detected the presence of many unusual types of bacterial flora. These tribal people do not have problems with diabetes, many forms of cancer and many diseases of civilization. Bacterial worlds came into existence well before humans evolved. When someone migrate to a western country from the tropical region, these bacteria have to make adjustments to the newer life style of their host and some of them may get replaced and others that could not adapt might not survive these adaptive changes of the host; consequently immunity could be weakened.

Stress may lead to the disequilibrium of bioenergy fields, which can result in disruption of immunity and disease. Theoretically, organisms can heal themselves through the mobilization of extrasomatic energy. While therapeutic intervention may not bring about direct healing, it may put into place the conditions needed for an organism can heal itself through leveraging its subtle energy. All healing, therefore, may ultimately involve self-healing. We know extraordinarily little about these hidden channels of the body, but many believe that they have great bearing in maintaining homeostasis and immunity. We may have hibernating energy resources that can be mobilized in such a way that any infection from a pathogen will be met with a secondary immune response that terminates the infection before it causes disease. For some, this may be seen as spiritual energy, and in these unprecedented and extraordinary circumstances, it may be a good idea to focus on extra-physiological factors to lift the immune powers, whatever that means to an individual. A holistic approach can be more effective than a unitary approach. Like environmental factors, extra-physiological factors may also contribute to the genesis of bodily ailments. Weakening of this hidden immune system can leave people vulnerable to infection but strengthening them can lead to better health.

\section{Conclusion}

COVID-19 might ultimately affect many people, but one way to defeat this silent enemy is to balance our defence systems. Those who have heightened immunity will be able to survive the disease with less physical damage and suffering and minimal symptoms; they may even be asymptomatic. It is a good thing that there is ample promotion of preventive measures of the contagion, there should be more awareness of improving personal immunity. More research works are warranted in immunology including extra-physiological immunity. COVID-19 has a direct impact on co-existing disease processes and the elderly people tend to have weakened immunity. The immune system of migrant people has to make several adaptations during the migration process, and this is particularly relevant o for those who migrate from tropical weather to cold weather. The deranged external and internal environment 
has weakened our collective immunity and made our individual body a playground for the coronavirus, so more attention should be focused on balance our immune systems to combat the COVID-19 virus. Humans did not have any resources to beat the epidemics in the pre-vaccine era. Nature intervened with its great healing powers in those earlier periods of history and many of the highprofile diseases faded from the public consciousness even without widespread vaccine use. Like humans have an extra-physiological immune mechanism, nature may have highly refined defense mechanisms to thwart pathogens. If that is the case, the Corona virus would make a truce with the nature at some point or the other way and remain in the background. In the years to come, COVID-19 would contribute new insights into virology.

\section{References}

1. Tan W, Zhao X, Ma X, Wang W, Niu P, et al. (2020) A novel coronavirus genome identified in a cluster of pneumonia cases-Wuhan, China 20192020. China CDC Weekly 2(4): 61-62.

2. Lu R, Zhao X, Li J, Niu P, Yang B, et al. (2020) Genomic characterization and epidemiology of 2019 novel coronavirus: implications for virus origins and receptor binding. The Lancet 395(10224): 565-574.

3. Blalock JE (1984) The immune system as a sensory organ. Journal of Immunology 132(3): 1067-1070.

4. Lynch L (2016) iNRT cells induce maximal weight loss in GLPI therapy. Cell Metabolism Cell Press 24(3): 510-519.

5. (2020) Excess weight can increase risk of serious illness and death from COVID-19. Public Health England.

6. Sureda A, Bibiloni M, Julibert A, Bouzas C, Argelich E, et al. (2020) Adherence to the mediterranean diet and inflammatory markers. Nutrients 10(1): 62 .

7. Grant WB, Lahore H, McDonnell SL, Baggerly CA, French CB, et al. (2020) Evidence that vitamin D supplementation could reduce risk of influenza and COVID-19 infections and deaths. Nutrients 12(4): 988.

8. Cartney DM, Byrne DG (2020) Optimisation of vitamin D status for enhanced immuno-protection against Covid-19. Ir Med J Apr 113(4): 58.

9. Jakovac H (2020) COVID-19 and vitamin D: is there a link and an opportunity for intervention? Am J Physiol Endocrinol Metab 318(5): E589.

10. (2020) ClinicalTrials.gov. COVID-19 and vitamin D supplementation: a multicentre randomized controlled trial of high dose versus standard dose vitamin D3 in high-risk COVID-19 patients (CoVitTrial).

11. Bzura BMG (2018) Vit D and influenza prevention or therapy? Int J Mol Sci 19(8): 2419.

12. Garland CF, Kim JJ, Mohr SB, Gorham DB, Grant WB, et al. (2014) Meta-analysis of all-cause mortality according to serum 25-hydroxyvitamin D. Am J Public Health 104(8): e43-e50.

13. Greiller CL, Martineau AR (2015) Modulation of the immune response to respiratory viruses by Vitamin D. Nutrients 7(6): 4240-4270.

14. Zakharova I, Klimov L, Kuryaninova V, Nikitina I, Malyavskaya S, et al. (2019) Vitamin d insufficiency in overweight and obese children and adolescents. Front Endocrinol 10: 103.

15. Nieman DC, Wentz LM (2019) The compelling link between physical activity and the body's defence system. J Sport Health Sci 8(3): 201-217.

16. Prather AA, Leung CW (2016) Association of insufficient sleep with respiratory infection among adults in the United States. JAMA Intern Med 176(6): 850-852.
17. Ohen S, Doyle WJ, Alper CM, Deverts DJ, Turner RB (2009) Sleep habits and susceptibility to the common cold. Archives of Internal Medicine 169(1): 62-67.

18. Westermann J, Lange T, Textor J, Born J (2015) System consolidation during sleep-A common principle underlying psychological and immunological memory formation. Trends in Neurosciences 38(10): 585-597.

19. Sarkar D, Jung MK, Wang HJ (2015) Alcohol and the immune system. Alcohol Res 37(2): 153-155.

20. Friedman H, Newton C, Klein TW (2003) Microbial infections, immunomodulation, and drugs of abuse. Clin Microbiol Rev 16(2): 209-219.

21. Vardavas CI, Nikitara K (2020) COVID-19 and smoking: a systematic review of the evidence. Tob Induc Dis 18: 20.

22. Tonnesen P, Marott JL, Nordestgaard B, Bojesen SE, Lange P (2019) Secular trends in smoking in relation to prevalent and incident smoking-related disease: A prospective population-based study. Tob Induc Dis 17: 72.

23. Zhou Z, Chen P, Peng H (2016) Are healthy smokers really healthy? Tob Induc Dis.

24. Liu W, Tao ZW, Lei W, Yuan ML, Liu K, et al. (2020) Analysis of factors associated with disease outcomes in hospitalized patients with 2019 novel coronavirus disease. Chin Med J 133(9).

25. Lippi G, Henry BM (2020) Active smoking is not associated with severity of coronavirus disease 2019 (COVID-19). Eur J Intern Med 75: 107-108.

26. Park JE, Jung S, Kim A (2018) MERS transmission and risk factors: a systematic review. BMC Public Health 18(1): 574.

27. Kokolus Km, Capitano Ml, Lee CT, Jason WL, Waight JD, et al. (2013) Metabolic cold stress accelerated tumour growth. Proceedings of the National Academy of Sciences 110(50): 20176-20181.

28. Mace TA, Zhong L, Kilpatrick C, Zynda E, Lee CT, et al. (2011) Differentiation of CD8+ T cells into effector cells is enhanced by physiological range hyperthermia. J Leukoc Biol 90(5): 951-962.

29. Federation of American Societies for Experimental Biology (2011) Elevated body temperature helps certain types of immune cells to work better, evidence suggests. Science Daily.

30. Caroline MLE, Danielle T, Pierre D (1998) Differential effects of temperature on specific and nonspecific immune defences in fish. J Exp Biol 201: 165-168.

31. Mailoo VJ, Chow G, Wilkins AMJ, Kennish S (2013) Psychoneuroimmunology of infection: implications for occupational therapy. International Journal of Therapy and Rehabilitation 18(11).

32. Andrew S (2014) Mind-body health and healing. Central Recovery Press, Nevada, USA.

33. Pandarakalam JP (2020) Interactions of quantum bioenergy fields. Neuro Quantology 18(2): 157-172.

34. Dispenza J (2014) You are the placebo. London: Hay House, USA.

35. Lockhart M (2010) The subtle energy body: The Complete Guide. Inner Traditions, Rochester, USA.

36. Collinge W (1998) Subtle energy. Warner Books, New York, USA.

37. Rubik B (1989) The interrelationship between mind and matter. The Center for Frontier sciences. Philadelphia, USA.

38. Rubik B (2002) Scientific analysis of the human aura. In: Heinze RI (Ed.), Proceedings of the $18^{\text {th }}$ International Conference on the Study of Shamanism and Alternative Modes of Healing. Santa Sabina Center, Dominican University, Calif, USA.

39. Krippner S, Rubin D (1974) The Kirlian aura. Anchor Books, New York, USA. 
40. Hansen JN, Lieberman JA (2013) Use of a torsion pendulum balance to detect and characterize what may be a human bioenergy field. Journal of Scientific Exploration 27(2): 205-225.

41. Tiller AW (1993) What are subtle energies? Journal of Scientific Exploration 7 (3): 293-304.
42. Keen JS (2018) The mind's interaction with the laws of physics and cosmology. Cambridge: Cambridge Scholars Publishing, USA.

43. Jenna M (2020) The science of staying well. Thorsons, London, UK.

For possible submissions Click below: 